This item was submitted to Loughborough's Research Repository by the author.

Items in Figshare are protected by copyright, with all rights reserved, unless otherwise indicated.

\title{
Exploitation of quasi-orthogonal space time block codes in virtual antenna arrays: part II Monte Carlo-based throughput evaluation
}

PLEASE CITE THE PUBLISHED VERSION

\section{PUBLISHER}

(C) IEEE

\section{VERSION}

VoR (Version of Record)

\section{LICENCE}

CC BY-NC-ND 4.0

\section{REPOSITORY RECORD}

Kassim, Shakiru K., Matthew Hayes, Nasr E.M. Eltayeb, and Jonathon Chambers. 2019. "Exploitation of Quasi-orthogonal Space Time Block Codes in Virtual Antenna Arrays: Part II Monte Carlo-based Throughput Evaluation". figshare. https://hdl.handle.net/2134/5386. 
This item was submitted to Loughborough's Institutional Repository (https://dspace.lboro.ac.uk/) by the author and is made available under the following Creative Commons Licence conditions.

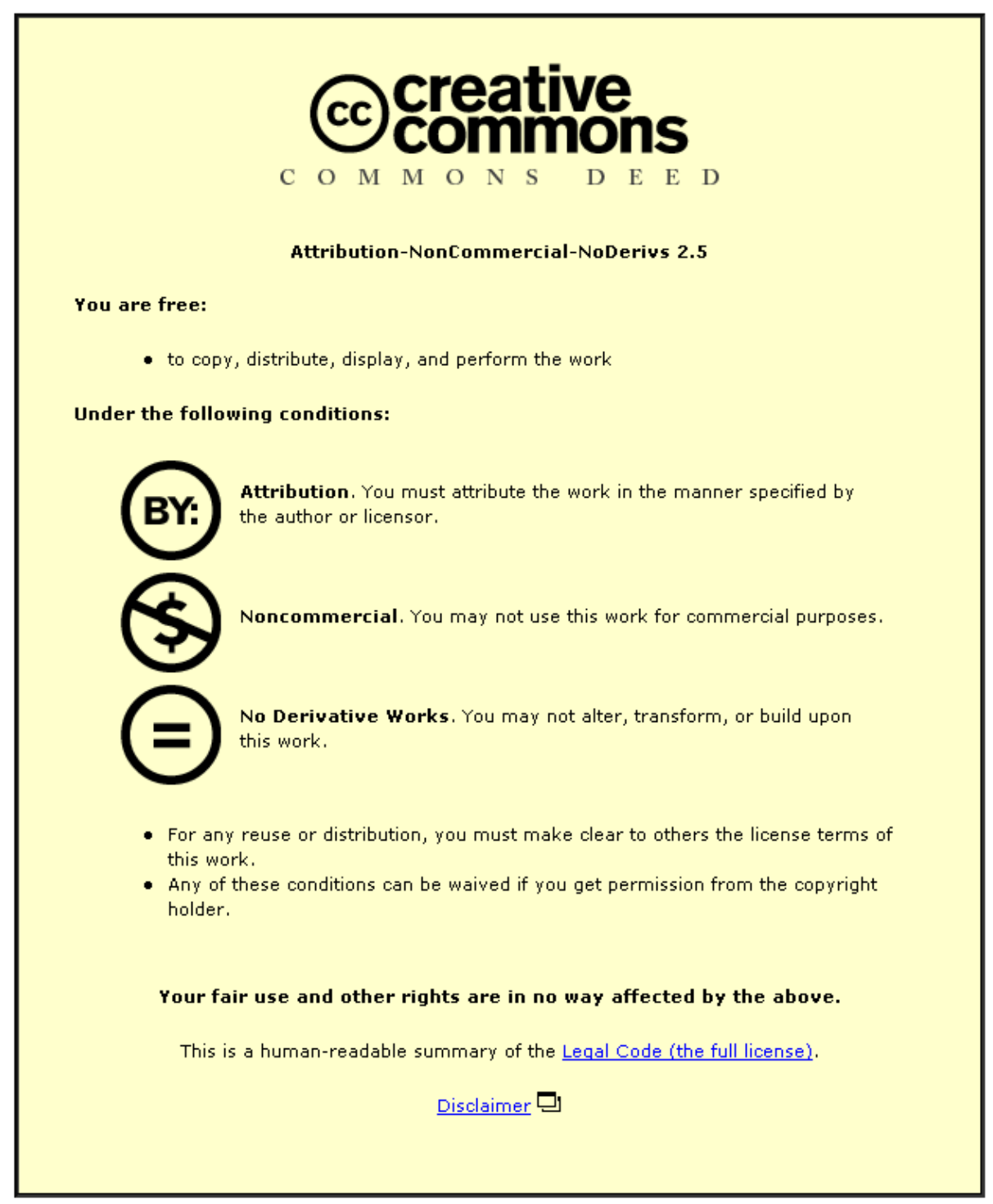

For the full text of this licence, please go to: http://creativecommons.org/licenses/by-nc-nd/2.5/ 


\title{
Exploitation of Quasi-Orthogonal Space Time Block Codes in Virtual Antenna Arrays: Part II Monte Carlo-based Throughput Evaluation
}

\author{
S.K.Kassim, M. Hayes, N.M. Eltayeb and J.A. Chambers \\ Advanced Signal Processing Group, Dept of Electronic and Electrical Engineering, Loughborough University, LE11 3TU, UK \\ Email: \{s.k.kassim,m.hayes,n.e.eltayeb,j.a.chambers\}@lboro.ac.uk
}

\begin{abstract}
A full rate and full diversity closed-loop quasiorthogonal space time block coding scheme due to Toker, Lambotharan and Chambers is proposed for application in virtual antenna arrays. The performance gain is achieved through closed-loop operation involving feedback of phase rotation angle(s) calculated from channel state information (CSI) to the transmitter array. Throughput performance of the proposed scheme, with and without power optimisation, is investigated through Monte Carlo simulation with QPSK constellation signals. The results confirm the improvement in throughput performance over orthogonal space time block codes.
\end{abstract}

Index Terms- Cooperative diversity, quasi-orthogonal space-time block codes (QOSTBCs), closed-loop phase angle feedback, multi-input multi-output (MIMO), virtual antenna arrays (VAAs)

\section{INTRODUCTION}

In next generation wireless communication systems there will be an increasing demand for wireless systems with high quality of service (QoS) and data capacity specification. Efforts have therefore been made to design wireless systems that offer such performance with the introduction of transmit diversity and receive diversity in multi-input multioutput (MIMO) systems which thereby offer robustness to the effect of fading in wireless channels [1]. Such systems generally yield higher capacity and hence better performance over conventional single-input single-output (SISO) systems. Research has also shown theoretically that as the number of transmit/receive antennas used in such systems increases so too does the capacity performance and the robustness of such MIMO systems. This motivates the introduction of closed-loop quasi-orthogonal space time block codes in [2] which use four transmit and $\mathrm{n}$ receive antennas and overcome the limitation of an earlier quasi-orthogonal space time block code introduced in [3] that expands the scheme introduced by Alamouti into four transmit and one receiver antenna system. However, a major constraint of such a system is how practical it is to ensure spatial uncorrelated sub-channels in a point-to-point MIMO system, since a high correlation among transmitting antennas potentially reduces a MIMO channel to a SISO channel. A solution to overcome this problem is to have an array of spatially distributed single antenna elements which form a virtual antenna array [4].

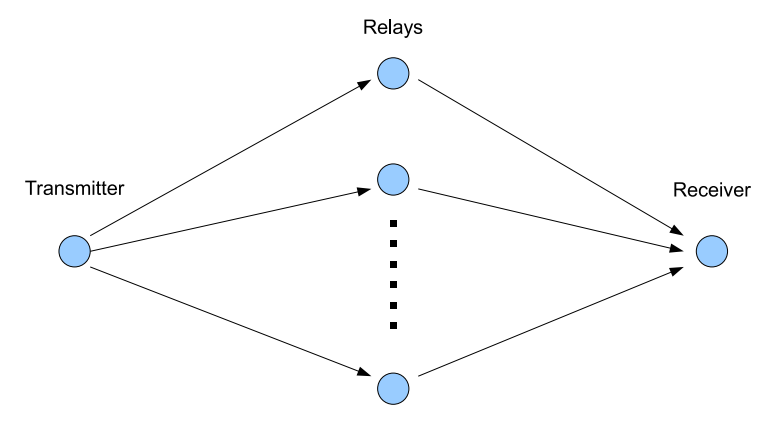

Fig. 1. Simple two-stage relay architecture, the relay stage would be formed from a set of mobile terminals.

Traditionally, a communication system is designed such that the base station communicates with each mobile terminal individually. Such a system as a whole, however, offers limited capacity and data throughput and can be expensive due to the infrastructure required. A more pragmatic approach to the system design would be to allow multi stage communication between the mobile terminals and the base stations which could be further enhanced if mutual communication between the mobile terminals is possible, creating a virtual MIMO channel [5] as shown in Fig.1. In this deployment, a VAA forms a special network which bridges the cellular and ad-hoc concepts to establish a heterogenous network with potentially increased capacity.

The work presented here is a novel idea that enhances the performance of the previously proposed multi stage VAA systems in [4]. It is demonstrated that with or without optimum power allocation between the relaying stages the total throughput of this system depends largely on the number of antennas deployed at each stage. However, with power constraint, the notion of effective power allocation between stages in a relaying network provides a significant advantage in the performance of the entire network. In this paper, it is 
assumed that the channels are ergodic, equal power allocation is employed within each stage, and the air interface between each VAA within a relaying stage is distinct from the interface used for inter-stage communication and is not optimised. It is also assumed that there is full cooperation among transmitting antennas at each relaying stage and the cooperative links are error free due to relatively short communication distance. Part 1 of this paper presented the theoretical end-to-end capacity of this system while here we concentrate upon Monte-Carlo simulations to verify the theoretical results.

The organization of this paper is as follows. Section II provides a brief review of the structure for the two stage relaying system using both space time block codes (STBC) and quasi-orthogonal STBCs (QOSTBC) along with the transmit antenna phase rotation based on feedback, and how power can be optimised between the stages. Simulation results are presented in Section III. Finally, Section IV provides the conclusion.

\section{RELAYING WITH STBCS AND QUASI-ORTHOGONAL STBCS}

\section{A. STBCs and Orthogonal STBCs for four transmit antennas}

In [6], it has been shown that full diversity and full code rate complex valued space-time block codes exist only for a dimension of two. An example is the well-known scheme due to Alamouti [1], which has the coding matrix

$$
C_{2 X 2}=\left[\begin{array}{cc}
c_{1} & c_{2} \\
-c_{2}^{*} & c_{1}^{*}
\end{array}\right]
$$

where $c_{i}, i=1,2$ represent the space time symbols and $(.)^{*}$ denotes complex conjugate. Likewise, an extension of this code first presented in [3] given below in equation (2), named as quasi-orthogonal space time block code (QO-STBC) provides partial diversity gain with full rate.

$$
C_{4 X 4}=\left[\begin{array}{cccc}
c_{1} & c_{2} & c_{3} & c_{4} \\
-c_{2}^{*} & c_{1}^{*} & -c_{4}^{*} & c_{3}^{*} \\
-c_{3}^{*} & -c_{4}^{*} & c_{1}^{*} & c_{2}^{*} \\
c_{4} & -c_{3} & -c_{2} & c_{1}
\end{array}\right]
$$

This is evidenced in the channel decoding matrix at the receiver. Suppose we transmit this scheme over a wireless channel (after taking the complex conjugates of the symbols in the second and third intervals) the received signal at the output over four symbol intervals can be expressed as

$$
\begin{gathered}
{\left[\begin{array}{l}
y_{1} \\
y_{2}^{*} \\
y_{3}^{*} \\
y_{4}
\end{array}\right]=\left[\begin{array}{cccc}
h_{1} & h_{2} & h_{3} & h_{4} \\
h_{2}^{*} & -h_{1}^{*} & h_{4}^{*} & -h_{3}^{*} \\
h_{3}^{*} & h_{4}^{*} & -h_{1}^{*} & -h_{2}^{*} \\
h_{4} & -h_{3} & -h_{2} & h_{1}
\end{array}\right]\left[\begin{array}{c}
c_{1} \\
c_{2} \\
c_{3} \\
c_{4}
\end{array}\right]+\left[\begin{array}{c}
n_{1} \\
n_{2}^{*} \\
n_{3}^{*} \\
n_{4}
\end{array}\right]} \\
Y=\mathbf{H C}+\mathbf{n}
\end{gathered}
$$

where, $Y$ is the received signal vector, $\mathbf{H}$ represents the transmission path matrix, $\mathbf{C}$ is the transmitted symbol vector and $\mathbf{n}$ contains the zero-mean circularly symmetric complex valued Gaussian noise components. Applying the matrix $\mathbf{H}^{H}$ to perform matched filtering, the output can then be expressed as

$$
\mathbf{Y}=\mathbf{H}^{H} Y
$$

where $(.)^{H}$ denotes Hermitian, complex conjugate, transpose.

Unlike Alamouti's scheme, which yields a diagonal $\mathbf{H}^{H} \mathbf{H}$ matrix with elements $\gamma=\Sigma_{n=1}^{t}\left|h_{n}\right|^{2}$, where t represents the number of transmitting antennas and $\left|h_{n}\right|^{2}$ is the magnitude squared of each transmission path, which demonstrates the full orthogonality of the code and hence produces a full diversity transmission, the QOSTBC $\mathbf{H}^{H} \mathbf{H}$ matrix is of the form

$$
H=\left[\begin{array}{cccc}
\gamma & 0 & 0 & \alpha \\
0 & \gamma & -\alpha & 0 \\
0 & -\alpha & \gamma & 0 \\
\alpha & 0 & 0 & \gamma
\end{array}\right]
$$

with some nonzero but equal off diagonal elements $\alpha$, which yield coupling between estimated symbols and hence reduce the diversity gain of the code. Toker et al. in [2] proposed and demonstrated two feedback methods that can be used to mitigate against this, i.e. to set $\alpha=0$, the first one is to phase rotate the transmitted symbols from two antennas with particular angles $\theta$ and $\phi$ such that

$$
\theta=\arccos \left(\frac{|\lambda|}{\kappa} \cos (\phi+\angle \lambda)\right)-\angle \kappa
$$

provided $\phi$ is in the range $\phi \epsilon[0,2 \pi)$ if $|\lambda|<|\kappa|$, where $\kappa=\Sigma_{n=1}^{t} h_{1}^{*} h_{4}$ and $\lambda=\Sigma_{n=1}^{t} h_{2}^{*} h_{3}$ with $|$.$| and \angle$. denoting absolute value and angle respectively. However, because of practical limitation it was further shown that using a single phase angle for rotation, (keeping the feedback from the receiver to the transmitter as small as possible) yields identical performance of the system [7]. Another method proposed was antenna weighting and selection, this involves pre-multiplying the transmitted symbols by a weighting diagonal matrix, which is determined by channel state information CSI feedback from the receiver, this helps to determine which two antennas are chosen to be weighted for higher performance. In this work the single phase angle approached is adopted as it requires minimum feedback information.

It should be noted that using the same number of receive antennas the full rate and full diversity QO-STBCs provide a higher diversity order i.e. fourth order, as compared to the conventional STBCs for two antennas, with second order diversity.

Each of these schemes is combined with a maximum ratio combiner (MRC) that utilised two or four receive antennas, in order to exploit the importance of optimum power allocation in a two stage relaying system.

\section{B. Symbol error rate SER and bit error rate BER analysis for QO-STBCs, STBCs and MRC Relaying Systems}

From equation (4) the instantaneous SNR $\rho$ per symbol $\mathbf{C}$ at the detection stage is given as

$$
\rho=\frac{1}{R} \frac{\lambda}{t} \frac{S}{N}
$$


where $S$ is the average transmit signal power, $N$ is the average received noise power, $R$ is the rate, $t$ is the number of transmit antennas and $\lambda \equiv\|\mathbf{H}\|^{2}$ where $\|.\|^{2}$ is a square Frobenius norm.

In this paper the schemes employed are all full rate, therefore $R$ is assumed to be unity. Since the instantaneous $\operatorname{SNR} \rho$ is random, due to the randomness of the channel realization $\lambda$, a given modulation scheme such as QPSK, therefore produces a probability of error conditioned on $\rho$ i.e. $P(\epsilon \mid \rho)$. The average probability is then expressed as

$$
P(\epsilon)=\int_{0}^{\infty} P(\epsilon \mid \rho) \cdot p d f_{\rho}(\rho) d \rho
$$

Expressing the integral in terms of the moment generating function (MGF) $\emptyset_{\rho}(s)$ of the instantaneous SNR which is expressed as

$$
\emptyset_{\rho}(s) \equiv \emptyset_{\frac{1}{R} \frac{\lambda}{t} \frac{S}{N}}(s)
$$

for coherent MPSK the probability of error (the average symbol error rate SER $P_{s}(\epsilon)$ can be expressed as

$$
P(\epsilon)=\frac{1}{\pi} \int_{0}^{\pi \frac{M-1}{M}} \emptyset_{\rho}\left(\frac{G P S K}{\sin ^{2} \theta}\right) d \theta
$$

where $M$ is the constellation size and GPSK $\equiv$ $\sin ^{2}(\pi / M)$.

The closed form of equation (10) for equal sub channel gain with MGF

$$
\emptyset_{\frac{1}{R} \frac{\lambda}{t} \frac{S}{N}}(s)=\frac{1}{\left(1-\frac{1}{R} \frac{\lambda}{t} \frac{S}{N} \cdot s\right)^{q}}
$$

where $\mathrm{s}$ is the number of symbols and $q \equiv t . r$ with $t$ and $r$ representing the number of transmit/received antennas respectively, is presented in [4].

The equivalent bit error rate $\operatorname{BER}\left(P_{b}(\epsilon)\right)$ expression is obtained when the bits are gray-mapped onto symbols i.e. adjacent symbol constellation points differ only by one bit and are thus expressed as

$$
P_{b}(\epsilon) \equiv \frac{P_{s}(\epsilon)}{\log _{2} M}
$$

at low BERs and SERs.

The probability of error expressed above estimates the average probability of a single relaying link, however the overall error performance of a given relaying system i.e. the end-to-end BER, which is expressed as a function of the number of relaying stages with $j$ transmit/recieve antennas at high BER can be expressed as

$$
P T(\epsilon)=1-\prod_{v=1}^{k}\left(1-P_{v}(\varepsilon)\right)
$$

where $k$ is the number of relaying stage which for this paper is limited to 2 and $P_{v}(\varepsilon)$ represents the BER of the $v^{\text {th }}$ stage.
In this work we investigate the above analysis using MonteCarlo simulations to produce the predicted end-to-end probability of error. This was investigated using regenerative relaying links for both STBC and QOSTBC. Given appropriate power limitation we evaluate power allocation strategies between the stages.

\section{Throughput and Power Allocation with QO-STBCs, STBCs and MRC Relaying Systems}

Throughput is a function of information delivered from source to sink; if we assume that the correctness of information is determined at the receiver.

To maximise the end-to-end throughput is the same as to minimise the end-to-end BER expressed in equation (14) below. If a source transmits $B$ bits/frame, where a frame is defined as $D=B / \log _{2} M$, to a target over k relaying stages, the normalised end-to-end throughput $\Theta$ can be expressed as

$$
\Theta=\min _{v \epsilon(1, k)}\left(\alpha_{v} R_{v} \log _{2}\left(M_{v}\right)\right) \cdot(1-P T(\epsilon))^{B}
$$

where $R_{v}$ is the STBC rate of the $v^{t h}$ stage, $M_{v}$ is the modulating index of the $v^{\text {th }}$ stage, $\alpha_{v}$ is the optimised frame duration of the $v^{t h}$ stage under the constraint that $\sum_{v=1}^{k} \alpha_{v}=1, P T(\epsilon)$ is the end-to-end BER and min signifies the dependence of throughput on the weakest link in the chain.

The end-to-end throughput is maximised by minimising the end-to-end BER through optimally assigning fractional power to each relaying stage with our assumption that the fractional frame duration is optimised.

The elegant analysis presented in [4] simplifies BER by invoking its upper bound which occurs when equation (10) is upper-bounded by its largest argument $\theta=\pi / 2$ and thus the presented BER of the $v^{t h}$ relaying stage for MPSK is bounded by

$$
P(\epsilon) \leq \sum_{v=1}^{K} \frac{M_{v}-1}{M_{v} \log _{2}\left(M_{v}\right)}\left(1+\beta_{v} \frac{G P S K}{R_{v}} \frac{\gamma_{v}}{t_{v}} \frac{S}{N}\right)^{-q}
$$

where $\gamma_{v}$ represents the average attenuation experienced at the $v^{t h}$ stage and $t_{v}$ is the number of transmit antennas of the $v^{t h}$ stage .

This upper bound was then utilised to determine the effective fractional allocating power $\beta_{v}$ that obeys

$$
\beta_{v} \approx\left[\alpha_{w}\left(\frac{q_{v}^{-1} A_{v}^{-1} B_{v}^{q_{v}}}{q_{w}^{-1} A_{w}^{-1} B_{w}^{q_{w}}}\right)^{\frac{1}{q_{\max }+1}}\right]^{-1}
$$

where $q_{\max }=\arg \max \left(q_{1}, \ldots ., q_{k}\right)$ and $w$ is the previous relaying stage and

$$
\begin{gathered}
A_{v}=\frac{M_{v}-1}{M_{v} \log _{2} M_{v}} \\
B_{v}=\frac{G P S K_{v}}{R_{v}} \frac{\gamma_{v}}{t_{v}} \frac{S}{N}
\end{gathered}
$$

The result presented next demonstrates the performance of a relaying system which exploits fully orthogonal QOSTBCs with the power allocation rule in equation (16) 


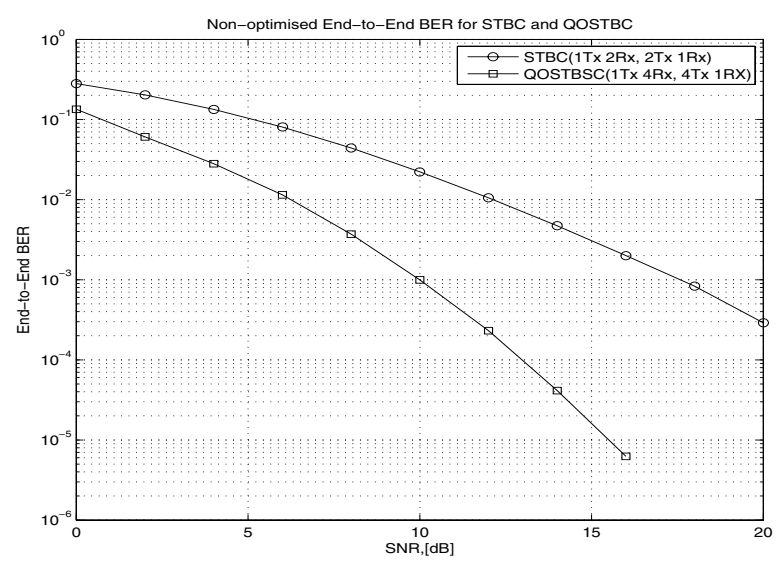

Fig. 2. End-to-end BER performance for an MRC, STBC and QOSTBC 2-stage relay with equal power allocation, with QPSK transmission.

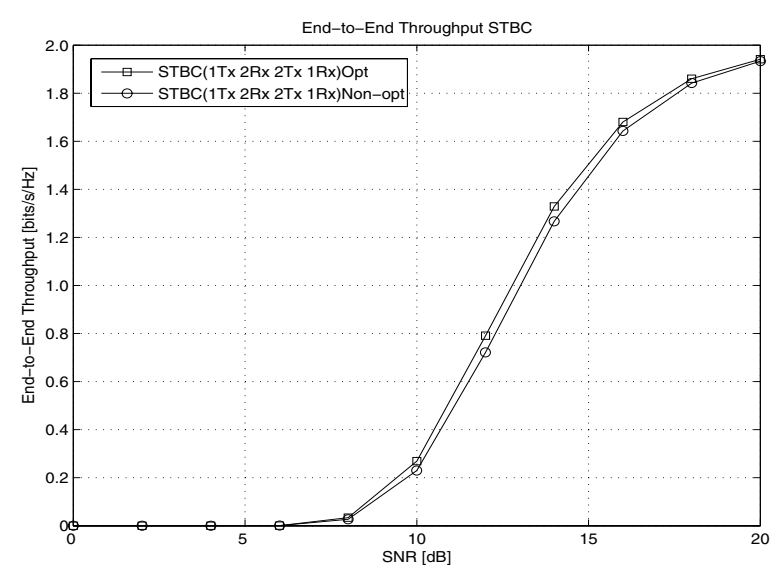

Fig. 4. End-to-end throughput performance for an MRC, STBC 2-stage relay with optimum and non-optimum power allocation.

\section{SIMULATION RESULTS}

In Figures 2 and 3, we show the end-to-end (average) BER performance of the proposed relaying systems with and without power optimisation for QPSK transmission. Only one antenna at the receiver was considered, but this technique can be extended to multiple receive antennas. The $\mathrm{x}$-axis shows the $\mathrm{SNR}$ in $\mathrm{dB}$ which is $3 \mathrm{~dB}$ above $E_{b} / N_{o}$ and the y-axis shows the end-to-end BER. In order to evaluate the BER performance, we considered ergodic flat fading channels. The end-to-end BER performance of the relaying system based on MRC and STBC performs worse when compared to the end-to-end BER performance of the relaying system based on MRC and QOSTBC for both optimum and non-optimum cases. In Figure 2 for example, an improvement of $7 \mathrm{~dB}$ is achieved with BER of $10^{-3}$ when a relaying system exploits full rate, full diversity QOSTBC. Likewise in Figure 3 a further improvement of $2 \mathrm{~dB}$ is gained when power allocations to the two stages are optimised. Hence it can be inferred

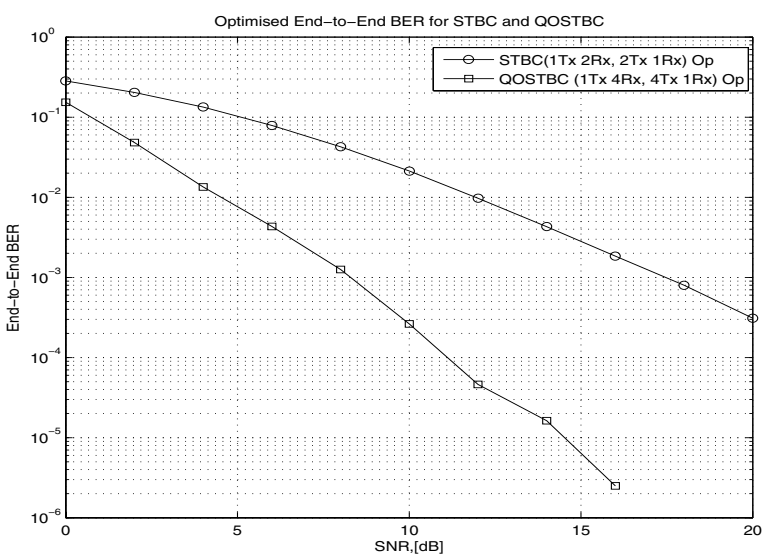

Fig. 3. End-to-end BER performance for an MRC, STBC and QOSTBC 2-stage relay with optimum power allocation, with QPSK transmission.

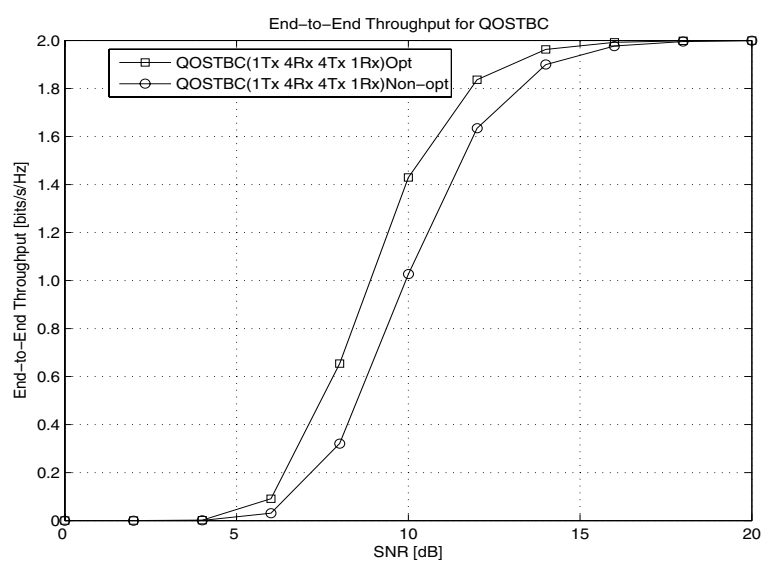

Fig. 5. End-to-end throughput performance for an MRC, QOSTBC 2-stage relay with optimum and non-optimum power allocation.

that deploying multiple antennas within a relaying system has the capacity to produce better bit error performance which is further enhanced if power is optimally allocated between relaying stages. These properties have the capability to increase the throughput of the entire system, Figures 4 and 5 make this more apparent.

When transmitting 100bits per frame, Figure 4 suggests that at SNR of $10 \mathrm{~dB}$, when attempting to transmit QPSK over the available channel, both optimum and non optimum power allocation account for loss of over $80 \%$ of the total transmitted frame with throughput less that 0.4 with MRC and STBC based relaying system. This suggests a limitation of this type of configuration (as evidenced in the BER performance). However, in Figure 5, a system containing a full rate, full diversity QOSTBC yields a throughput of either 1.0 approximately, nonoptimised, or 1.4, optimised at 10dB SNR. This confirms the advantage of the proposed scheme and in addition the gain in performance resulting from power optimisation. 


\section{CONCLUSION}

In summary, the results presented support the potential advantage of deploying a multiple antenna relaying system, with appropriate power allocation between the stages, to improve the performance of such networks although, with additional complexity.

We presented the performance comparison for two and four antenna relaying systems with STBC and closed-loop QOSTBC, the performance clearly shows a $7 \mathrm{~dB}$ gain when a four antenna relaying system is deployed as compared with the two antenna relaying system. Crucially, even with quantised feedback this advantage would be retained and the performance would be no worse than a two antenna relaying system if the feedback is completely lost. This significant improvement in performance further suggests a huge amount of power saving at the expense of the complexity of a four antenna system over a two antenna system. We further investigated the effect of optimum power allocation between the two stages of both systems, our findings confirmed that optimal power allocation between the stages in both STBC and closed-loop QOSTBC further increased the performance (although the increase depends on the numbers of antennas) with the effect more pronounced in the closed-loop QOSTBC relaying network.

In practice the system presented is a distributed processing system, as such, issues such as synchronisation, optimum power allocation within relaying stages, interference reduction, deployment of optimal space time code in a non traditional MIMO context, optimum number of cooperative antennas within relaying tiers and optimum numbers of relaying stages in order to preserve resources are still active research issues.

\section{ACKNOWLEDGMENT}

The authors wish to acknowledge the support of Dr M. Dohler and that of the Engineering and Physical Sciences Research Council of the U.K and QinetiQ Group Plc.

\section{REFERENCES}

[1] S.M. Alamouti, A simple transmit diversity technique for wireless communications, IEEE J.Select.Areas Commun., Vol. 16, pp.1451 - 1458, Oct. 1998.

[2] C. Toker, S. Lambotharan, and J.A. Chambers, Closed-Loop QuasiOrthogonal STBCs and Their Performance in Multipath Fading Environments and When Combined With Turbo Codes IEEE Trans. Wireless Communications, Vol. 3, pp. 1890-1896, Nov. 2004.

[3] H. Jafarkhani, A Quasi-Orthogonal Space-Time Block Code, IEEE Trans. Commun., Vol. 49, pp.1-4, Jan. 2001.

[4] M.Dohler, Virtual Antenna Arrays, PhD Thesis, University of London. Nov. 2003.

[5] L. Le and E. Hossain, Multihop Cellular Networks: Potential Gains, Research Challenges, and a Resource Allocation Framework, IEEE Communication Magazine, Vol.45, pp.66 - 73, Sept. 2007.

[6] V.Tarokh, H. Jafarkhani, and A.R. Calderbank, Space Time Block Codes from Orthogonal Designs, IEEE Trans. on Information Theory, Vol.45, pp.1456 - 1467, July 1999.

[7] S. Lambotharan, and C. Toker, Closed-Loop Space Time Block Coding Techniques for OFDM based Broadband Wireless Access System, IEEE Trans. on Consumer Electronics, Vol.51, pp.765 - 769, Aug. 2005. 